\title{
Hunting for Organic Molecules with Artificial Intelligence: Molecules Optimized for Desired Excitation Energies
}

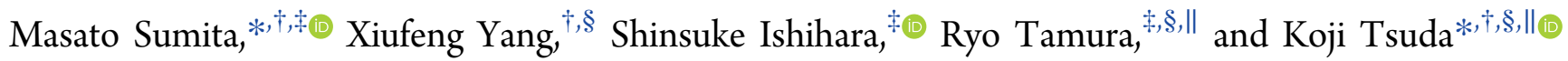 \\ ${ }^{\dagger}$ Center for Advanced Intelligence Project, RIKEN, 1-4-1 Nihombashi, Chuo-ku, Tokyo 103-0027, Japan \\ ${ }^{\ddagger}$ International Center for Materials Nanoarchitectonics (WPI-MANA), National Institute for Materials Science, 1-1 Namiki, \\ Tsukuba, Ibaraki 305-0044, Japan \\ ${ }^{\S}$ Graduate School of Frontier Sciences, The University of Tokyo, 5-1-5 Kashiwa-no-ha, Kashiwa, Chiba 277-8561, Japan \\ "Research and Services Division of Materials Data and Integrated System, National Institute for Materials Science, 1-2-1 Sengen, \\ Tsukuba, Ibaraki 305-0047, Japan
}

Supporting Information

ABSTRACT: This work presents a proof-of-concept study in artificial-intelligence-assisted (AI-assisted) chemistry where a machine-learning-based molecule generator is coupled with density functional theory (DFT) calculations, synthesis, and measurement. Although deep-learning-based molecule generators have shown promise, it is unclear to what extent they can be useful in real-world materials development. To assess the reliability of AI-assisted chemistry, we prepared a platform using a molecule generator and a DFT simulator, and attempted to generate novel photofunctional molecules whose lowest excited states lie at desired energetic levels. A 10 day run on the 12 -core server discovered 86 potential photofunctional molecules around target lowest excitation levels, designated as

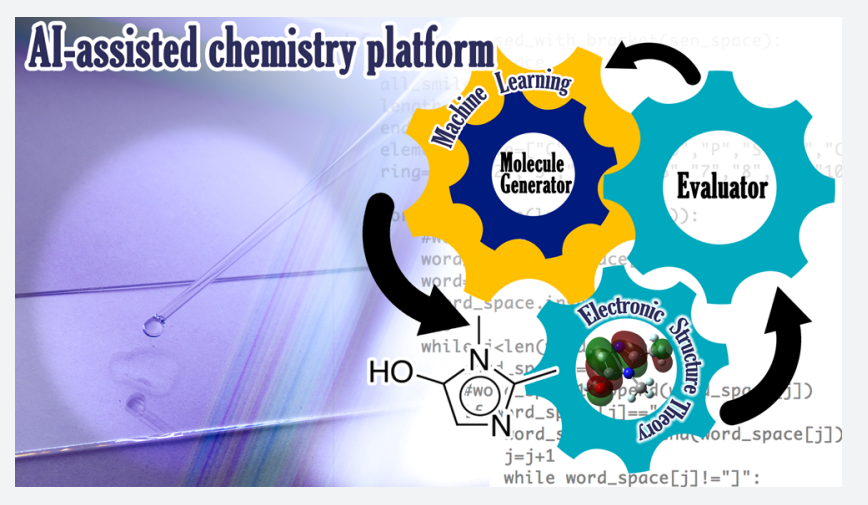
200, 300, 400, 500, and $600 \mathrm{~nm}$. Among the molecules discovered, six were synthesized, and five were confirmed to reproduce DFT predictions in ultraviolet visible absorption measurements. This result shows the potential of AI-assisted chemistry to discover ready-to-synthesize novel molecules with modest computational resources.

\section{INTRODUCTION}

The idea of using artificial intelligence (AI) for molecule design has existed for a long time but has never been fully realized. Although earlier attempts employing relatively simple methods such as heuristic enumeration ${ }^{1}$ and genetic algorithms ${ }^{2}$ yielded some success, these methods rely on arbitrary chemical rules. Stimulated by recent breakthroughs in deep learning, ${ }^{3}$ a new generation of de novo molecule design algorithms emerged and showed remarkable ability to generate functional molecules without chemical rules. ${ }^{4-8}$ Similar machine-learning algorithms have been used in designing inorganic materials as well. ${ }^{9-12}$ Figure 1 illustrates our AI-assisted chemistry platform to develop new molecules. It generates a large number of molecules using the loop of a machine-learning-based molecule generator and a quantum chemical package such as GAUSSIAN, ${ }^{13}$ GAMESS, ${ }^{14}$ or NWChem. ${ }^{15}$ It has been shown repeatedly that these methods can generate simulator-qualified molecules, i.e., molecules that are predicted to have the desired properties by a simulator. To what extent this can be useful to real-world materials development remains, however, largely unknown.

In this work, we conducted a proof-of-concept study to evaluate whether or not an AI-assisted chemistry platform can discover synthesizable, functional molecules in a reasonable computational time. As a testbed, we chose photofunctional organic molecules, which have received particular attention in green chemistry and molecular sensing. In photofunctional molecules, light induces transition between electronic states. Controlling the level of excited states of the molecules from their ground states is a common issue for organic electronics (like organic light-emitting diodes, ${ }^{16,17}$ organic photovoltaic cells $\left.{ }^{18,19}\right)$, photofunctional sensors, ${ }^{20}$ and UV filters. ${ }^{21}$

Our platform, consisting of ChemTS (a molecule generator) ${ }^{4}$ and a calculator (B3LYP/3-21G*) based on density functional theory (DFT), ${ }^{22}$ was configured to generate molecules whose first excited state is at five different wavelengths. A 10 day $(240 \mathrm{~h})$ run of our machine-learning algorithm on a 12-core (Intel Xeon E5-2689v3 CPU) server created a variety of molecules whose DFT-based wavelength was approximately at the desired value. Among them, six molecules were synthesized, and five of them were experimentally confirmed to have the desired wavelength, using ultraviolet visible (UV-vis) spectroscopy. This result

Received: April 8, 2018

Published: August 20, 2018 


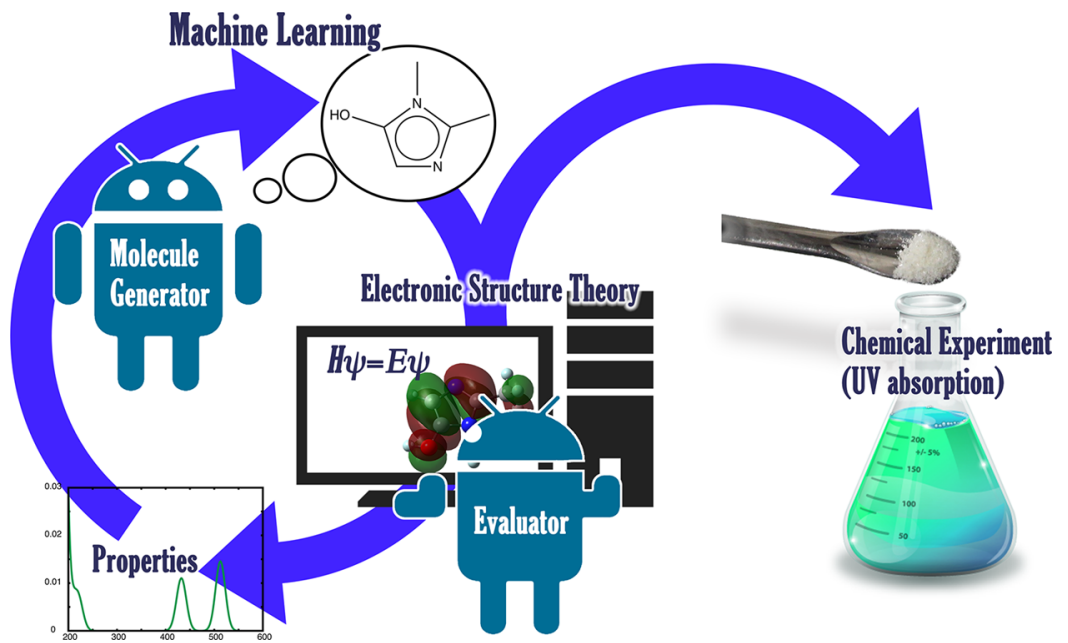

Figure 1. Our AI-assisted chemistry platform for discovering new functional molecules. The Android robot is reproduced or modified from work created and shared by Google and used according to terms described in the Creative Commons 3.0 Attribution License.

shows that the molecules generated by an AI-assisted platform have a high chance of being synthesizable and functional.

As exemplified by AlphaGO, ${ }^{23}$ an interesting aspect of $\mathrm{AI}$ is that it often finds unconventional ways to solve a problem. Our origin-of-excitation analysis of the synthesized molecules showed that our platform preferred $n-\pi^{*}$ excitation over $\pi-\pi^{*}$ excitation, conventionally used to control the wavelength. ${ }^{24,25}$ This illustrates AI-chemistry's ability to not only accelerate discovery, but also shed light on hidden paths of possible research.

\section{RESULTS AND DISCUSSION}

Our platform was configured to find molecules whose first excited states lie at 200, 300, 400, 500, and $600 \mathrm{~nm}(6.2-2.1 \mathrm{eV})$. The recurrent neural network in ChemTS was trained a priori with 13000 molecules. For each target wavelength, our platform ran for 2 days $(48 \mathrm{~h})$. The total numbers of molecules generated are summarized in Table 1 (the molecules included in ChemTS's

Table 1. Number of Molecules at Different Qualification Levels for Each Target Wavelength

\begin{tabular}{lrrrrc} 
& \multicolumn{5}{c}{ Target wavelength } \\
\cline { 2 - 6 } & $200 \mathrm{~nm}$ & $300 \mathrm{~nm}$ & $400 \mathrm{~nm}$ & $500 \mathrm{~nm}$ & $600 \mathrm{~nm}$ \\
Generated $^{a}$ & 646 & 757 & 629 & 607 & 638 \\
Simulator-qualified $^{b}$ & 34 & 26 & 13 & 12 & 1 \\
Synthesized $^{c}$ & 2 & 2 & 1 & 1 & 0 \\
Functional $^{c}$ & 1 & 2 & 1 & 1 & 0
\end{tabular}

${ }^{a_{\text {The }}}$ first row indicates the number of molecules generated by ChemTS. ${ }^{b}$ The second row shows the number of simulator-qualified molecules whose absorption wavelength is predicted by DFT to be within $20 \mathrm{~nm}$ error from the target. ${ }^{c}$ The third and fourth rows denote the number of synthesized molecules, and those experimentally confirmed by UV-vis measurement, respectively.

training set are not counted) as SMILES strings. ${ }^{26}$ Out of about 3200 molecules, 86 were found to be within $\pm 20 \mathrm{~nm}$ of the desired wavelength through DFT calculation (Table 2). The six molecules marked with roman numerals (I-VI) were selected as synthesizable molecules according to the following criteria: (1) At least one synthetic route is reported in SciFinder. ${ }^{27}$ (2) Oscillator strength obtained with time-dependent DFT (TD-DFT) is strong enough to allow the transition from the ground state to the first excited state.
UV-Vis Spectra Measurement. Figure 2 shows the results of UV-vis spectra measurement of $\mathbf{I}-\mathbf{V I}$, together with computational spectra at the B3LYP/3-21G* level. Except for II, the first peak (be it a shoulder or an edge of the peak) in each experimental spectrum lies close to the target wavelength. Note that solvatochromic effects in I-VI were small (see the Supporting Information).

We investigated the reason why molecule II failed to reproduce the DFT prediction. The broad peak around $350 \mathrm{~nm}$ is most likely caused by decomposition, as we observed trace impurity signals in the ${ }^{1} \mathrm{H}$ NMR spectrum taken after several weeks after synthesis (see the Supporting Information). Another possible cause is keto-enol tautomerization. According to the ${ }^{1} \mathrm{H}$ NMR measurement, the keto-form exists as a major peak (Figure S1 in the Supporting Information). The keto-form is more stable than II (enol-form) by $71.72 \mathrm{~kJ} \mathrm{~mol}^{-1}$ at the B3LYP/3-21G* level (Table S1 in the Supporting Information). Although the spectrum is definitely affected by keto-enol tautomerization, it does not seem to cause the absorption around $350 \mathrm{~nm}$, since the computational spectrum of the keto-form also failed to reproduce the peak (Figure S17 in the Supporting Information).

For molecule VI, we observed an unpredicted large peak from 500 to $300 \mathrm{~nm}$. The ${ }^{1} \mathrm{H}$ NMR spectrum of VI indicates that a tautomer in enol-form exists (Figure S14 in the Supporting Information). Each tautomer can have syn/anti conformers. As shown in Table S2 of the Supporting Information, the four isomers syn-keto, syn-enol, anti-keto, and anti-enol have small energetic differences, and can hence coexist. Among these isomers, only molecule VI (i.e., anti-/syn-keto) has a peak around $500 \mathrm{~nm}$ in its computational spectrum (Figure S18 in the Supporting Information), indicating that the edge at $500 \mathrm{~nm}$ is indeed due to molecule VI. These observations strongly suggest that the coexistence of four isomers of VI results in the large peak.

Origin of Excitation. Kohn-Sham orbitals involved in the first excited state of I-VI are summarized in Figure 3. A conventional means to control absorption wavelength focuses on a $\pi-\pi^{*}$ transition: the length of a $\pi$-system is altered to change the energy difference between $\pi$ and $\pi^{*}$ orbitals. ${ }^{24,25}$ Our AI-assisted platform seems to have taken a different approach: 10 out of 24 molecules (randomly sampled from 86 molecules in Table 2) 
Table 2. Simulator-Qualified Molecules ${ }^{a}$ as SMILES Strings Found by Our AI-Assisted Chemistry Platform

\begin{tabular}{|c|c|}
\hline SMILES & Wavelength / nm \\
\hline \multicolumn{2}{|l|}{ Target wavelength : $200 \mathrm{~nm}$} \\
\hline & $207.83\left(\mathrm{n}-\pi^{*}\right)$ \\
\hline \multicolumn{2}{|l|}{ Ce1ocen1 } \\
\hline $\mathrm{NC}(\mathrm{CCCHN}) \mathrm{O}$ & $187.90\left(\pi-\sigma^{*}\right)$ \\
\hline $\mathrm{OCNN} / \mathrm{C}=\mathrm{N} / \mathrm{O}$ & 214.61 \\
\hline $\mathrm{OC} 1=\mathrm{NCC} 2(\mathrm{C} 1) \mathrm{CCCC} 2$ & 210.64 \\
\hline $\mathrm{CNC}[\mathrm{C} @ @ \mathrm{H}](\mathrm{C}(=\mathrm{O}) \mathrm{O}) \mathrm{O}$ & 216.14 \\
\hline $\mathrm{N}[\mathrm{C} @ @ \mathrm{H}](\mathrm{C}[\mathrm{C} @ \mathrm{H}](\mathrm{CC}(\mathrm{C}) \mathrm{C}) \mathrm{O}) \mathrm{Cc} 1 \mathrm{ccco} 1$ & $218.76\left(n-\pi^{*}\right)$ \\
\hline Cc1onc(c1)O & 200.19 \\
\hline $\mathrm{N}[\mathrm{C} @ \mathrm{H}](/ \mathrm{C}(=\mathrm{NO}) / \mathrm{O}) \mathrm{CCC}$ & 217.61 \\
\hline ON1CC1 & 191.69 \\
\hline O[C@H]([C@@H]1CCNCC1)N(C) & 189.79 \\
\hline $\mathrm{NC}[\mathrm{C} @ \mathrm{H}] 1 \mathrm{OC}[\mathrm{C} @ \mathrm{H}]([\mathrm{C} @ \mathrm{H}]([\mathrm{C} @ \mathrm{H}] 1 \mathrm{O}) \mathrm{C}) \mathrm{O}$ & $212.47\left(\pi-\sigma^{*}\right)$ \\
\hline N[C@@H]([C@@H](CC(O)C)O)Cc1 cnc[nH]1 & 203.28 \\
\hline C1OCN1CN1CCOCC1 & 202.96 \\
\hline O[C@@H]([C@H]([C@H](CN)C)O)ON(CC)CC & $219.52\left(n-\sigma^{*}\right)$ \\
\hline $\mathrm{N}[\mathrm{C} @ \mathrm{H}](\mathrm{CCN} 1 \mathrm{CCNCC} 1) \mathrm{C}$ & 197.73 \\
\hline $\mathrm{N}[\mathrm{C} @ \mathrm{H}](\mathrm{CC} \# \mathrm{CC}(\mathrm{C}) \mathrm{C}) \mathrm{O}$ & 185.70 \\
\hline OCCCCN(CCO)C[C@@H](O)C & 184.41 \\
\hline $\mathrm{ON}=\mathrm{C}(\mathrm{O}) \mathrm{C}$ & $205.47\left(\pi-\sigma^{*}\right)$ \\
\hline $\mathrm{C} / \mathrm{C}=\mathrm{N} / \mathrm{N} 1 \mathrm{CC}[\mathrm{C} @ \mathrm{H}](\mathrm{C} 1) \mathrm{O}$ & 211.44 \\
\hline $\mathrm{C} / \mathrm{C}=\mathrm{N} / \mathrm{N}[\mathrm{C} @ \mathrm{H}] 1 \mathrm{CCCCO} 1$ & 207.96 \\
\hline $\mathrm{NC}(\mathrm{C})(\mathrm{C}) \mathrm{C}$ & 180.70 \\
\hline ONCCC[C@H](CC(C)C)O & $185.83\left(n-\sigma^{*}\right)$ \\
\hline C1OCN1 & 204.52 \\
\hline $\mathrm{O}[\mathrm{C} @ @ \mathrm{H}] 1 \mathrm{CN} 2 \mathrm{CC}[\mathrm{C} @ \mathrm{H}] 1 \mathrm{CC} 2$ & 185.49 \\
\hline & $207.42(n-\pi *)$ \\
\hline \multicolumn{2}{|l|}{ Ce1nec(n1C)O } \\
\hline C1NCCOCC1 & 182.76 \\
\hline $\mathrm{CCON} / \mathrm{C}(=\mathrm{NC}) / \mathrm{O}$ & $218.04\left(\pi-\sigma^{*}\right)$ \\
\hline OC[C@@H](NC[C@H](O)C)O & 187.37 \\
\hline OC[C@@H](OCCCN(C)C)C & 183.27 \\
\hline $\mathrm{OC}[\mathrm{C} @ @ \mathrm{H}]([\mathrm{C} @ \mathrm{H}]([\mathrm{C} @ @ \mathrm{H}]([\mathrm{C} @ @ \mathrm{H}](\mathrm{O}) \mathrm{C}(=\mathrm{N}) \mathrm{O}) \mathrm{O}) \mathrm{O}) \mathrm{O}$ & 188.61 \\
\hline $\mathrm{NN} 1 \mathrm{C}(=\mathrm{N}) \mathrm{OC}[\mathrm{C} @ \mathrm{H}] 1 \mathrm{C}$ & $181.22\left(\mathrm{n}-\pi^{*}\right)$ \\
\hline $\mathrm{O}[\mathrm{C} @ \mathrm{H}] 1 \mathrm{C}[\mathrm{C} @ \mathrm{H}] 2 \mathrm{C}([\mathrm{C} @ @ \mathrm{H}](\mathrm{C} 1) \mathrm{N} 2 \mathrm{C}) \mathrm{O}$ & 195.73 \\
\hline $\mathrm{C}=\mathrm{C}[\mathrm{C} @ @ \mathrm{H}] 1 \mathrm{CCC}(=\mathrm{N} 1) \mathrm{O}$ & 213.01 \\
\hline C1OC[C@@H]2N(C1)CCO2 & 187.11 \\
\hline \multicolumn{2}{|l|}{ Target wavelength: $300 \mathrm{~nm}$} \\
\hline $\mathrm{N}[\mathrm{C} @ @ \mathrm{H}] 1 \mathrm{C}(=\mathrm{O})[\mathrm{C} @ @] 2(\mathrm{C}([\mathrm{C} @ \mathrm{H}] 1 \mathrm{CC} 2)(\mathrm{C}) \mathrm{C}) \mathrm{C}$ & $299.81\left(\mathrm{n}-\pi^{*}\right)$ \\
\hline $\mathrm{N} \# \mathrm{Cc} 1 \mathrm{c}(\mathrm{OC}) \mathrm{cc}[\mathrm{nH}] \mathrm{c} 1=\mathrm{O}$ & 300.7 \\
\hline $\mathrm{C} / \mathrm{N}=\mathrm{C}(/ \mathrm{O}[\mathrm{N}][\mathrm{CH}] \mathrm{c} 1 \mathrm{ccc}(\mathrm{cc} 1) \mathrm{OC}) \mathrm{O}$ & 282.13 \\
\hline
\end{tabular}


Table 2 . continued

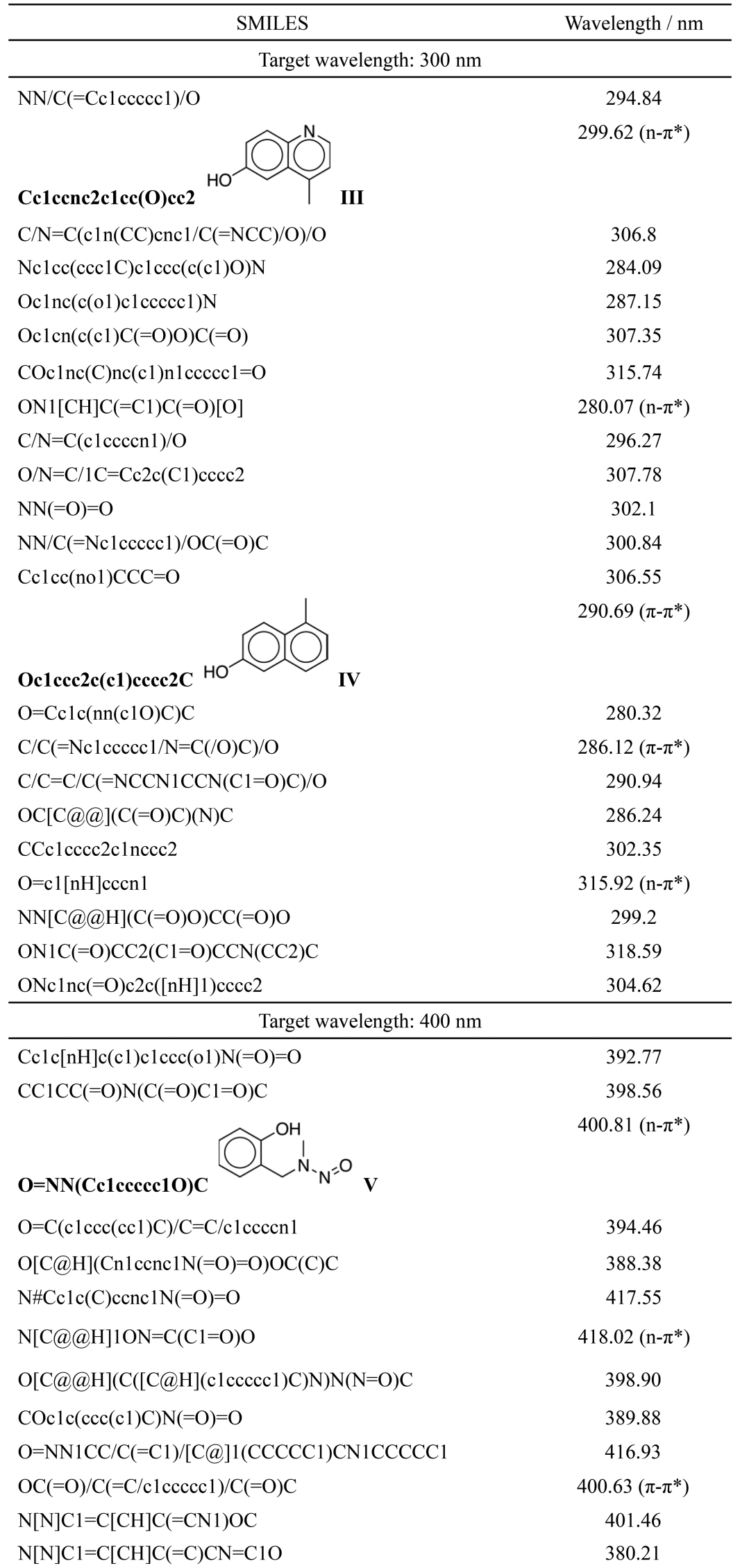


Table 2. continued

\begin{tabular}{|c|c|}
\hline SMILES & Wavelength / nm \\
\hline \multicolumn{2}{|c|}{ Target wavelength: $500 \mathrm{~nm}$} \\
\hline & $484.43\left(\mathrm{n}-\pi^{*}\right)$ \\
\hline \multicolumn{2}{|l|}{$\mathbf{C C}(=\mathbf{O}) \mathbf{C}(=\mathbf{O}) \mathbf{C N}(\mathbf{C}) \mathrm{C} \quad \mathrm{O} \quad \mathrm{VI}$} \\
\hline$[\mathrm{O}] \mathrm{N} 1[\mathrm{CH}] \mathrm{Cc} 2 \mathrm{c}(\mathrm{C} 1) \operatorname{ccc} 2$ & $480.46\left(\pi-\pi^{*}\right)$ \\
\hline$[\mathrm{O}][\mathrm{N}] \mathrm{N} 1[\mathrm{CH}] \mathrm{N}=\mathrm{C}([\mathrm{N}] 1) \mathrm{NN}(=\mathrm{O})=\mathrm{O}$ & 483.83 \\
\hline$[\mathrm{O}][\mathrm{N}] \mathrm{N}(\mathrm{c} 1 \mathrm{ccccc} 1) \mathrm{C}(=\mathrm{O}) \mathrm{c} 1 \mathrm{ccccc} 1$ & 487.75 \\
\hline$[\mathrm{O}][\mathrm{N}] \mathrm{O} / \mathrm{C}(=\mathrm{NCC}) / \mathrm{N}$ & 484.53 \\
\hline$[\mathrm{O}][\mathrm{N}] \mathrm{O} / \mathrm{C}=\mathrm{N} / \mathrm{c} 1 \mathrm{ccccc} 1$ & $500.24\left(\pi-\pi^{*}\right)$ \\
\hline$[\mathrm{O}][\mathrm{N}] \mathrm{O} / \mathrm{C}(=\mathrm{NCC}) / \mathrm{O}$ & 500.24 \\
\hline$[\mathrm{O}][\mathrm{N}] \mathrm{N} 1[\mathrm{CH}] \mathrm{N}=\mathrm{C}([\mathrm{N}] 1) \mathrm{NN}(=\mathrm{O})=\mathrm{O}$ & 489.31 \\
\hline$[\mathrm{O}][\mathrm{N}] \mathrm{N} 1[\mathrm{CH}] \mathrm{N}=\mathrm{C}([\mathrm{N}] 1) \mathrm{N}$ & 486.36 \\
\hline$[\mathrm{O}][\mathrm{N}] \mathrm{N} 1[\mathrm{CH}] \mathrm{N}=\mathrm{C}([\mathrm{N}] 1) \mathrm{O}$ & $487.37\left(\mathrm{n}-\pi^{*}\right)$ \\
\hline$[\mathrm{O}] \mathrm{N}(\mathrm{N}(\mathrm{c} 1 \mathrm{cccc} 1)[\mathrm{O}]) \mathrm{c} 1 \mathrm{cccc}(\mathrm{c} 1) \mathrm{N}$ & 484.17 \\
\hline$[\mathrm{O}][\mathrm{N}] \mathrm{N} 1[\mathrm{C} @ @ \mathrm{H}](\mathrm{CCN}=\mathrm{C} 1 \mathrm{O}) \mathrm{Cc} 1 \mathrm{ccccc} 1$ & 482.01 \\
\hline \multicolumn{2}{|c|}{ Target wavelength: $600 \mathrm{~nm}$} \\
\hline $\mathrm{O}=\mathrm{Nn} 1 \mathrm{c}(\mathrm{O}) \mathrm{nccc} 1=\mathrm{O}$ & $606.58\left(\mathrm{n}-\pi^{*}\right)$ \\
\hline
\end{tabular}

${ }^{a}$ The synthesized molecules are shown with their chemical structural formula. Randomly sampled 24 molecules' origins of excitation are shown in parentheses with excitation wavelengths.
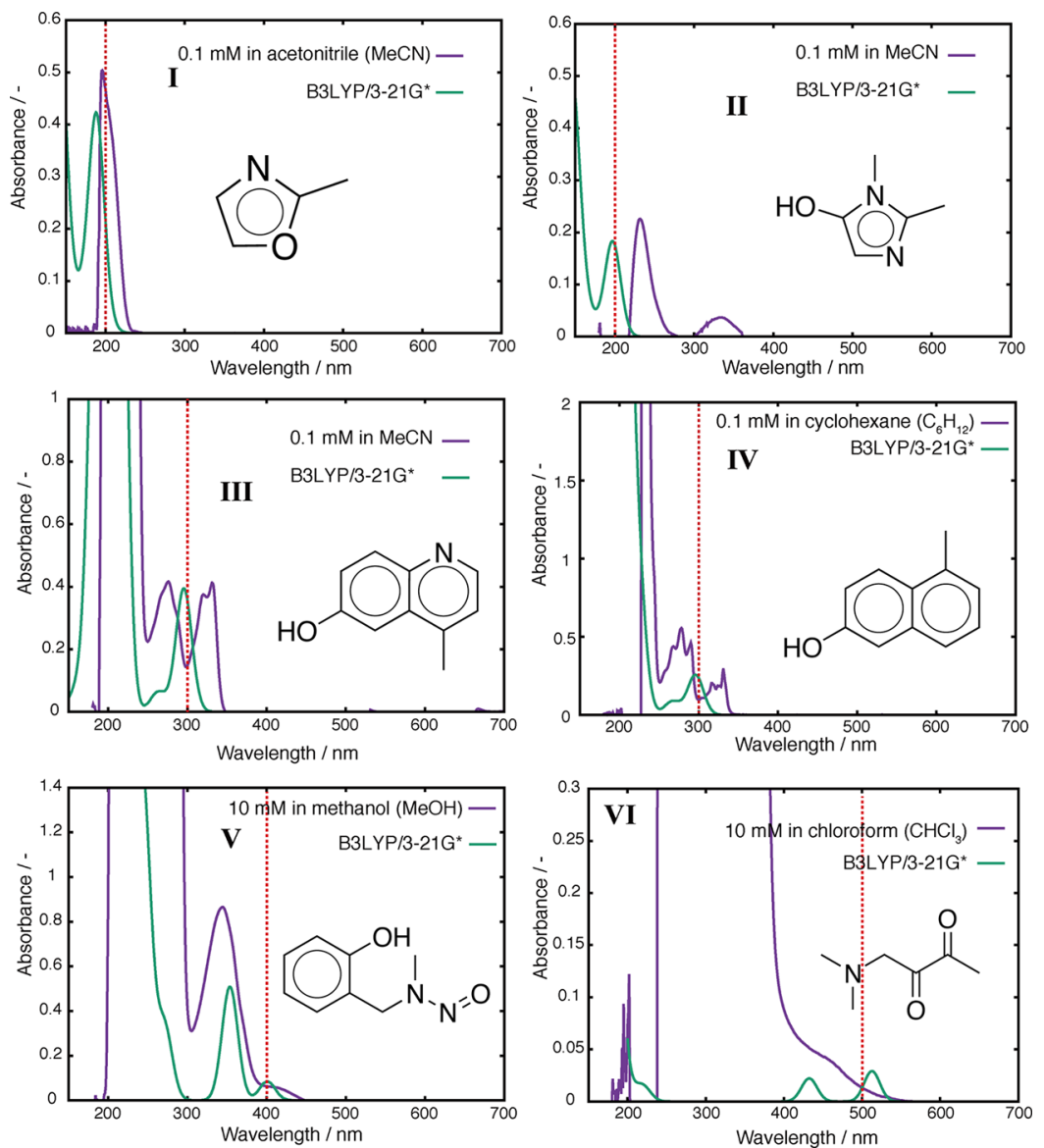

Figure 2. Experimental UV-vis absorption spectra and computational spectra at the B3LYP/3-21G* level of the compounds I-VI. The computational spectra are smoothed by a Gaussian function and arbitrarily scaled for comparison with the experimental spectra. The red dashed line in each spectrum indicates the target wavelength. 


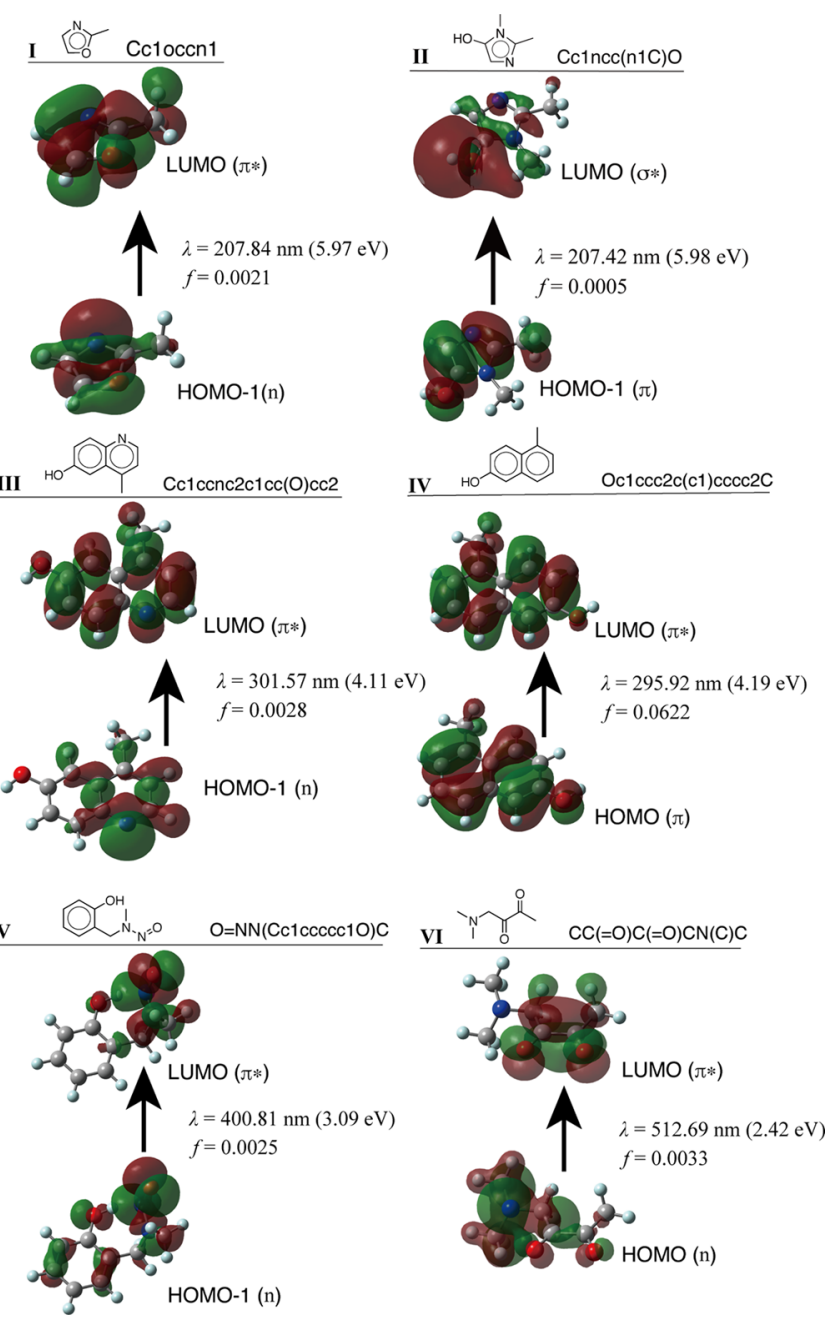

Figure 3. Main Kohn-Sham orbitals involved in the first excited states of I-VI at the B3LYP/3-21G* level. HOMO and LUMO denote the highest occupied molecular orbital and the lowest unoccupied molecular orbital, respectively. $\lambda$ and $f$ denote the computational absorption wavelength and oscillator strength, respectively.

show an $n-\pi^{*}$ transition (41\%), and 5 out of 24 molecules show a $\pi-\pi^{*}$ transition (21\%). For molecules I, III, V, and VI, the first excited state corresponds to an $n-\pi^{*}$ transition. Only molecule IV is associated with a $\pi-\pi^{*}$ transition. Interestingly, the failed molecule II is based on a $\pi-\sigma^{*}$ transition.

The lowest excitation energy of molecule $\mathbf{I}$ is exceptionally high $(207.84 \mathrm{~nm})$. Typically, $\mathrm{n}-\pi^{*}$ transitions have lower excitation energy than $\pi-\pi^{*}$, because an ordinary nonbonding orbital lies between $\pi$ and $\pi^{*}$ orbitals in energy. For example, the absorption bands of the $n-\pi^{*}$ transition of azobenzene derivatives appear around 400-600 $\mathrm{nm}$ in UV-vis spectra. ${ }^{24,28}$ It is likely that $\sigma$ orbital mixing stabilized the nonbonding orbital of nitrogen to lie lower in energy than a $\pi$ orbital.

From the shape of orbitals in Figure 3, the transitions on molecules III, V, and VI indicate charge transfer. Under charge transfer, TD-DFT with conventional hybrid functionals often underestimates the excitation energy due to self-interaction error. ${ }^{29}$ Fortunately, in the present instance, the error caused by charge transfer was limited, but it might become an issue in other types of molecule design problems.

Molecule II is the only one with a $\pi-\sigma^{*}$ excitation. Since $\pi-\sigma^{*}$ excitations in aromatic molecules with $\mathrm{XH}(\mathrm{X}=\mathrm{N}, \mathrm{O}, \mathrm{S})$

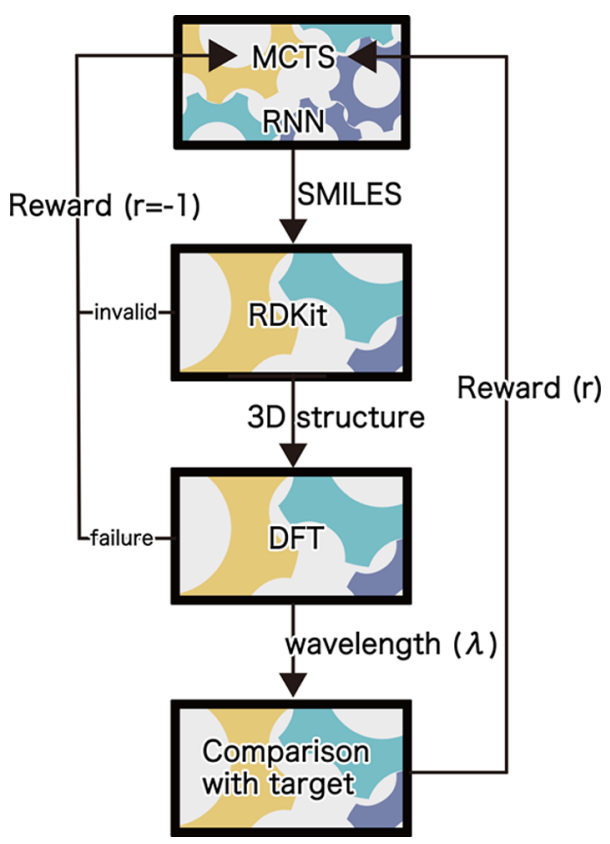

Figure 4. Workflow of a molecule generator $\left(\mathrm{ChemTS}^{4}\right)$ coupled with electronic structure theory (DFT). Molecules in SMILES string generated by ChemTS (MCTS+RNN) are converted to those in a three-dimensional structure with RDKit. ${ }^{36}$ Then, the computation for each molecule is performed with electronic structure theory to obtain the value of $\lambda$ (absorption wavelength). Reward $(r)$ is calculated by eq 1 . Rewards of molecules whose wavelengths are not available because of the failure in DFT calculation are set to -1 . The calculated reward $(r)$ reflects MCTS as back-propagation.

are reported as repulsive along the $\mathrm{X}-\mathrm{H}$ coordinate, ${ }^{30}$ we could predict that molecule II is extremely unstable to light, as was subsequently verified by the detection of decomposed products in the ${ }^{1} \mathrm{H}$ NMR spectrum (Figure S3 in the Supporting Information).

\section{CONCLUSION}

In this work we built a proof-of-concept study for an AI-chemistry platform, which was able to find five synthesizable and stable organic molecules possessing target properties within 10 days: a remarkable and encouraging result. Additionally, our platform exhibited the counterintuitive and intriguing tendency to use $n-\pi^{*}$ excitations. Since our platform depends on DFT calculation, it inherits its drawbacks: our analysis of failed cases, including tautomerization, isomers, and instability, shows the type of issues that future AI-chemistry platforms will have to overcome. In the near future, such platforms may be used in various molecule discovery projects, with the potential to change the landscape of chemistry research.

\section{METHODS}

Molecule Generator. We used the ChemTS library ${ }^{4}$ for searching for novel molecules with desired absorption wavelength. It generates molecules by using Monte Carlo Tree Search $(\mathrm{MCTS})^{31}$ and recurrent neural network (RNN). ${ }^{32,33}$ Figure 4 describes details of our workflow. Before the start, the RNN is trained with a set of SMILES strings. In our case, 13000 molecules that contain only $\mathrm{H}, \mathrm{O}, \mathrm{N}$, and $\mathrm{C}$ elements obtained from the PubChemQC database ${ }^{34,35}$ were used.

ChemTS generates one SMILES string at a time. In a normal round, the SMILES string is converted to a three-dimensional 
chemical structure by RDKit; the absorption wavelength $(\lambda)$ is computed by DFT, and the reward $(r)$ is calculated by the following equation

$$
r=\frac{-\alpha\left|\lambda^{*}-\lambda\right|}{1+\alpha\left|\lambda^{*}-\lambda\right|}
$$

where $\lambda^{*}$ indicates the target wavelength. Parameter $\alpha$ is set to 0.01 in this work. Using the reward information, ChemTS updates its parameters to generate better molecule in the next rounds. When the procedure fails because of an invalid SMILES string or DFT failure, $r=-1$ is fed back to ChemTS. Note that the following SMILES symbols are used: $\{\mathrm{C},[\mathrm{C} @ @ \mathrm{H}],(, \mathrm{N}$,$) ,$ $\mathrm{O},=, 1, /, \mathrm{c}, \mathrm{n},[\mathrm{nH}],[\mathrm{C} @ \mathrm{H}], 2,[\mathrm{NH}],[\mathrm{C}],[\mathrm{CH}],[\mathrm{N}],[\mathrm{C} @$ @], [C@], o, [O], 3, \#, [O-], [n+], [N+], [CH2], [n]\}.

Electronic Structure Theory. Relative to machine-learning algorithms, computation with electronic structure theory is very computationally costly. Therefore, we adopted density functional theory (DFT) with a well-known hybrid functional, B3LYP, taking into account the balance between reliability and computational costs. In addition, a $3-21 \mathrm{G}^{*}$ basis set was used to explore molecules efficiently in the chemical space. In the present work, we evaluated valence excited states of molecules, avoiding haphazard use of diffuse functions to exclude Reydberg states. To evaluate the excitation energy, we adopted timedependent DFT (TD-DFT) for the molecule generator at the aforementioned level. The lowest 20 states of each molecule were calculated after geometry optimization. All DFT calculations were performed with the Gaussian 16 package. ${ }^{13}$

UV-Vis Spectra Measurement. Electronic absorption spectra were measured using a Shimadzu UV-3600 UV-visNIR spectrophotometer at $20^{\circ} \mathrm{C}$. A quartz cell with $1 \mathrm{~cm}$ optical length was used. Spectroscopic grate solvents were purchased from Tokyo Chemical Industry (TCI) and Wako Pure Chemical Industries, and were used as received.

\section{ASSOCIATED CONTENT}

\section{S Supporting Information}

The Supporting Information is available free of charge on the ACS Publications website at DOI: 10.1021/acscentsci.8b00213.

Details and characterization of materials with ${ }^{1} \mathrm{H}$ NMR, DFT discussion of keto-enol tautomers of compounds II and VI, and investigation of dependence of solvent and concentration of each material (PDF)

\section{AUTHOR INFORMATION}

\section{Corresponding Authors}

*E-mail: masato.sumita@riken.jp.

*E-mail: tsuda@k.u-tokyo.ac.jp.

\section{ORCID}

Masato Sumita: 0000-0002-3506-1028

Shinsuke Ishihara: 0000-0001-6854-6032

Koji Tsuda: 0000-0002-4288-1606

\section{Notes}

The authors declare no competing financial interest.

Safety statement: no unexpected or unusually high safety hazards were encountered.

\section{ACKNOWLEDGMENTS}

Ms. Kumiko Hara is acknowledged for assisting in the measurement of absorption spectra. We also thank Kazuhiko Nagura, Atsuro Takai, Jinzhe Zhang, and David duVerle for the useful discussions. This work was supported by the "Materials Research by Information Integration” Initiative (MI2I) project and Core Research for Evolutional Science and Technology (CREST) [Grants JPMJCR1502 and JPMJCR17J2] from Japan Science and Technology Agency (JST). It was also supported by Grant-in-Aid for Scientific Research on Innovative Areas "Nano Informatics" [Grant 25106005] from the Japan Society for the Promotion of Science (JSPS). In addition, it was supported by Ministry of Education, Culture, Sports, Science and Technology (MEXT) as "Priority Issue on Post-K Computer" (Building Innovative Drug Discovery Infrastructure through Functional Control of Biomolecular Systems). The computations in this work were carried out on the supercomputer centers of NIMS and RAIDEN of AIP (RIKEN).

\section{REFERENCES}

(1) Gani, R.; Brignole, E. A. Molecular Design of Solvents for Liquid Extraction Based on UNIFAC. Fluid Phase Equilib. 1983, 13, 331-340.

(2) Venkatasubramanian, V.; Chan, K.; Caruthers, J. M. Evolutionary Design of Molecules with Desired Properties Using the Genetic Algorithm. J. Chem. Inf. Model. 1995, 35, 188-195.

(3) LeCun, Y.; Bengio, Y.; Hinton, G. Deep Learning. Nature 2015, 521, 436.

(4) Yang, X.; Zhang, J.; Yoshizoe, K.; Terayama, K.; Tsuda, K. ChemTS: an Efficient Python Library for De Novo Molecular Generation. Sci. Technol. Adv. Mater. 2017, 18, 972-976.

(5) Ikebata, H.; Hongo, K.; Isomura, T.; Maezono, R.; Yoshida, R. Bayesian Molecular Design with a Chemical Language Model. J. Comput.-Aided Mol. Des. 2017, 31, 379-391.

(6) Gómez-Bombarelli, R.; et al. Automatic Chemical Design Using a Data-Driven Continuous Representation of Molecules. ACS Cent. Sci. 2018, 4, 268-276.

(7) Kusner, M. J.; Paige, B.; Hernández-Lobato, J. M. Grammar Variational Autoencoder. In Proceedings of 34th International Conference on Machine Learning, ICML 2017; 2017; pp 1945-1954.

(8) Segler, M. H.; Kogej, T.; Tyrchan, C.; Waller, M. P. Generating Focused Molecule Libraries for Drug Discovery with Recurrent Neural Networks. ACS Cent. Sci. 2018, 4, 120-131.

(9) Pilania, G.; Balachandran, P. V.; Kim, C.; Lookman, T. Finding New Perovskite Halides via Machine Learning. Front. Mater. 2016, 3, 19.

(10) Liu, Y.; Zhao, T.; Ju, W.; Shi, S. Materials Discovery and Design Using Machine Learning. J. Materiomics 2017, 3, 159-177.

(11) Kim, C.; Pilania, G.; Ramprasad, R. Machine Learning Assisted Predictions of Intrinsic Dielectric Breakdown Strength of ABX3 Perovskites. J. Phys. Chem. C 2016, 120, 14575-14580.

(12) Faber, F. A.; Lindmaa, A.; von Lilienfeld, O. A.; Armiento, R. Machine Learning Energies of 2 Million Elpasolite $\left(\mathrm{ABC}_{2} \mathrm{D}_{6}\right)$ Crystals. Phys. Rev. Lett. 2016, 117, 135502.

(13) Frisch, M. J.; Trucks, G. W.; Schlegel, H. B.; Scuseria, G. E.; Robb, M. A.; Cheeseman, J. R.; Scalmani, G.; Barone, V.; Petersson, G. A.; Nakatsuji, H.; Li, X.; Caricato, M.; Marenich, A. V.; Bloino, J.; Janesko, B. G.; Gomperts, R.; Mennucci, B.; Hratchian, H. P.; Ortiz, J. V.; Izmaylov, A. F.; Sonnenberg, J. L.; Williams-Young, D.; Ding, F.; Lipparini, F.; Egidi, F.; Goings, J.; Peng, B.; Petrone, A.; Henderson, T.; Ranasinghe, D.; Zakrzewski, V. G.; Gao, J.; Rega, N.; Zheng, G.; Liang, W.; Hada, M.; Ehara, M.; Toyota, K.; Fukuda, R.; Hasegawa, J.; Ishida, M.; Nakajima, T.; Honda, Y.; Kitao, O.; Nakai, H.; Vreven, T.; Throssell, K.; Montgomery, J. A., Jr.; Peralta, J. E.; Ogliaro, F.; Bearpark, M.; Heyd, J. J.; Brothers, E. N.; Kudin, K. N.; Staroverov, V. N.; Kobayashi, R.; Normand, J.; Raghavachari, K.; Rendell, A.; Burant, J. C.; Iyengar, S. S.; Tomasi, J.; Cossi, M.; Millam, J. M.; Klene, M.; Adamo, C.; Cammi, R.; Ochterski, J. W.; Martin, R. L.; Morokuma, K.; Farkas, O.; Foresman, J. B.; Fox, D. J. Gaussian 16, revision A.03; Gaussian, Inc.: Wallingford CT, 2016.

(14) Schmidt, M. W.; et al. A. General Atomic and Molecular Electronic Structure System. J. Comput. Chem. 1993, 14, 1347-1363. 
(15) Valiev, M.; et al. NWChem: A Comprehensive and Scalable Open-Source Solution for Large Scale Molecular Simulations. Comput. Phys. Commun. 2010, 181, 1477-1489.

(16) Baldo, M. A.; et al. Highly Efficient Phosphorescent Emission from Organic Electroluminescent Devices. Nature 1998, 395, 151154.

(17) Kaji, H.; et al. Purly Organic Electroluminescent Material Realizing 100\% Conversion from Electricity to Light. Nat. Commun. 2015, 6, 8476.

(18) Li, Y. Molecular Design of Photovoltaic Materials for Polymer Solar Cells: Toward Suitable Electron Energy Levels and Broad Absorption. Acc. Chem. Res. 2012, 45, 723-733.

(19) Mazzio, K. A.; Luscombe, C. K. The Future of Organic Photovoltaics. Chem. Soc. Rev. 2015, 44, 78-90.

(20) Beer, P. D.; Gale, P. A. Anion Recognition and Sensing: the State of the Art and Future Perspectives. Angew. Chem., Int. Ed. 2001, 40, $486-516$.

(21) Shaath, N. A. Ultraviolet Filters. Photochem. Photobiol. Sci. 2010, 9, 464-469.

(22) Parr, R. G.; Yang, W. Density-Functional Theory of Atoms and Molecules; Oxford University Press: New York, 1989.

(23) Silver, D.; et al. Mastering the Game of Go without Human Knowledge. Nature 2017, 550, 354-359.

(24) Vollhardt, K. P. C.; Schore, N. E. Delocalized Pi Systems. In Organic Chemistry Structure and Function, 3nd ed.; W. H. Freeman and Company: New York, 1998; pp 583-636.

(25) Jones, R. N. The Ultraviolet Absorption Spectra of Aromatic Hydrocarbons. Chem. Rev. 1943, 32, 1-46.

(26) Weininger, D. SMILES a Chemical Language and Information System. 1. Introduction to Methodology and Encoding Rules. J. Chem. Inf. Model. 1988, 28, 31-36.

(27) SciFinder; Chemical Abstracts Service: Columbus, OH (accessed Oct 31, 2017); https://scifinder.cas.org.

(28) Samanta, S.; et al. Photoswitching Azo Compounds in vivo with Red Light. J. Am. Chem. Soc. 2013, 135, 9777-9784.

(29) Dreuw, A.; Head-Gordon, M. Failure of Time-Dependent Density Functional Theory for Long-Range Charge-Transfer Excited States: The Zincbacteriochlorin-Bacteriochlorin and Bacteriochlorophyll-Spheroidene Complexes. J. Am. Chem. Soc. 2004, 126, 40074016.

(30) Lim, J. S.; Choi, H.; Lim, I. S.; Par, S. B.; Lee, Y. S.; Kim, S. K. Photodissociation Dynamics of Thiophenol- $d_{1}$ : The Nature of Excited Electronic States along the S-D Bond Dissociation Coordinate. J. Phys. Chem. A 2009, 113, 10410-10416.

(31) Browne, C.; Powley, E.; Whitehouse, D. A Survey of Monte Carlo Tree Search Methods. IEEE Transactions on Computational Intelligence and $A I$ in Games 2012, 4, 1-43.

(32) Hochreiter, S.; Schmidhuber, J. Long Short-Term Memory. Neural Computation 1997, 9, 1735-1780.

(33) Cho, K., et al. Learning Phrase Representations Using RNN Encoder-Decoder for Statistical Machine Translation. In Proceedings of the 2014 Conference on Empirical Methods in Natural Language Processing, EMNLP 2014; 2014; pp 1724-1734.

(34) Public Computational Chemistry Database Project. http:// pccdb.org (accessed Nov 7, 2017).

(35) Nakata, M.; Shimazaki, T. PubChemQC Project: A Large-Scale First-Principles Electronic Structure Database for Data-Driven Chemistry. J. Chem. Inf. Model. 2017, 57, 1300-1308.

(36) Landrum, G. RDKit: Open-source cheminformatics. http:// www.rdkit.org (accessed Nov 7, 2017). 